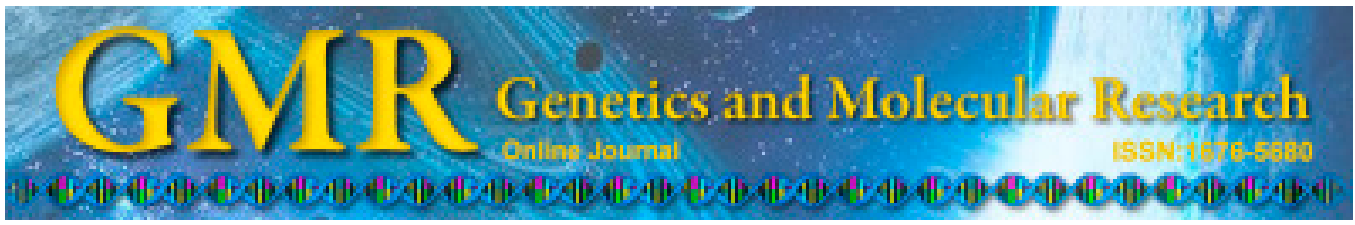

Short Communication

\title{
Genetic linkage map of Cucurbita maxima with molecular and morphological markers
}

\author{
Y. Ge ${ }^{1,2 *}$, X. Li ${ }^{1 *}$, X.X. Yang ${ }^{1}$, C.S. Cui ${ }^{1}$ and S.P. $Q u^{1}$ \\ ${ }^{1}$ Key Laboratory of Biology and Genetic Improvement of Horticultural Crops \\ (Northeast Region), Department of Horticulture, Northeast Agricultural University, \\ Harbin, China \\ ${ }^{2}$ Haikou Experimental Station, Chinese Academy of Tropical Agricultural Sciences, \\ Haikou, China \\ *These authors contributed equally to this study. \\ Corresponding author: S.P. Qu \\ E-mail: shupingqu@hotmail.com / qushuping1973@gmail.com
}

Genet. Mol. Res. 14 (2): 5480-5484 (2015)

Received November 10, 2014

Accepted March 26, 2015

Published May 22, 2015

DOI http://dx.doi.org/10.4238/2015.May.22.18

\begin{abstract}
Cucurbita maxima is one of the most widely cultivated vegetables in China and exhibits distinct morphological characteristics. In this study, genetic linkage analysis with 57 simple-sequence repeats, 21 amplified fragment length polymorphisms, 3 random-amplified polymorphic DNA, and one morphological marker revealed 20 genetic linkage groups of $C$. maxima covering a genetic distance of $991.5 \mathrm{cM}$ with an average of $12.1 \mathrm{cM}$ between adjacent markers. Genetic linkage analysis identified the simple-sequence repeat marker 'PU078072' $5.9 \mathrm{cM}$ away from the locus ' $R c$ ', which controls rind color. The genetic map in the present study will be useful for better mapping, tagging, and cloning of quantitative trait loci/gene(s) affecting economically important traits and for breeding new varieties of C. maxima through marker-assisted selection.
\end{abstract}

Key words: Cucurbita maxima; Genetic linkage map; Rind color; Molecular and morphological markers 


\section{INTRODUCTION}

The genus Cucurbita is composed of several important cultivated species. Three of these species, Cucurbita moschata, C. pepo, and C. maxima, are economically important crops worldwide (Robinson and Decker-Walters, 1997). C. maxima is thought to have originated in the Republic of Bolivia, southern Peru, and northern Argentina in South America (Whitaker and Davies, 1962; Esquinas-Alcazar and Gulick, 1983; Sanjur et al., 2002).

In recent decades, several genetic maps of the genus Cucurbita have been constructed with different parental species, including a map that used interspecific crosses between C. ecuadorensis and C. maxima (Weeden and Robinson, 1986), 2 maps using interspecific crosses between C. moschata and C. pepo (Lee et al., 1995; Brown and Myers, 2002), 4 intraspecific crosses of $C$. реро (Zraidi et al., 2007; Gong et al., 2008a; Esteras et al., 2012), and 1 intraspecific cross of $C$. moschata (Gong et al., 2008b), but no maps of an intraspecific cross of C. maxima have been published for the economically important genus Cucurbita (C. moschata, C. maxima, and C. pepo).

The published genetic maps of the genus Cucurbita have used different kinds of markers, mapping populations crossed from different parental species, to contribute to identifying quantitative trait loci for fruit shape and the depth of indentations between primary leaf veins (Brown and Myers, 2002) and 10 quantitative traits (Esteras et al., 2012). Moreover, these published genetic maps also helped in locating the loci controlling qualitative traits for turning yellow before anthesis, silver mottling of leaves, and the intensity of rind color (Brown and Myers, 2002), silver mottling of leaves, naked seeds, and bush growth habit (Zraidi et al., 2007), naked seeds and bush growth habit (Gong et al., 2008a), naked seeds and green rind (Gong et al., 2008b), and 12 qualitative traits such as vine, immature fruit, and mature fruit (Esteras et al., 2012). However, there are no published molecular genetic studies for orange and gray rind colors.

The objective of the present study was to construct a genetic linkage map of $C$. maxi$m a$ using random-amplified polymorphic DNA (RAPD), amplified fragment length polymorphism (AFLP), simple-sequence repeats (SSRs), and 1 phenotypic marker to locate the locus controlling rind color. The map of C. maxima can be used in further studies for identifying the loci controlling qualitative and quantitative traits.

\section{MATERIAL AND METHODS}

The $201 \mathrm{~F}_{2}$ mapping population was derived by crossing 2 diverse $C$. maxima lines '982-351' and '06820-1' from the College of Horticulture, Northeast Agricultural University. The maternal parent (98-2-351) was an inbred line with a gray rind. The paternal parent (06820-1) was an inbred line with an orange rind (Figure 1). The seedlings were cultivated in each row with a distance of $0.5 \mathrm{~m}$ between seedlings in the experimental field of Northeast Agriculture University in China. Cultivation management was in accordance with standardized agronomic procedures.

Rind color (orange and gray) as a qualitative trait was controlled by 1 gene and orange rind was dominant to gray rind ( $\mathrm{Li}, 2014)$. Rind color (orange and gray) were measured visually relative to the mature fruits of the mapping population, $\mathrm{F}_{1}$, and 2 parental lines.

A total of 1386 SSR primer pairs with the prefix "CMTm" and "PU" (Gong et al., 2008a; http://www.icugi.org/), 256 AFLP EcoRI and MseI primer combinations (Li, 2014), and 50 RAPD primer pairs with the prefix "S" (Li, 2014) were screened for polymorphisms between the parental lines '98-2-351' and '06820-1'. DNA extraction was conducted according to the modified sodium dodecyl sulfate procedure (Ge et al., 2011). Polymerase chain reaction amplification, separation, and observation of samples were conducted as described previously (Ge et al., 2012; Li, 2014). 

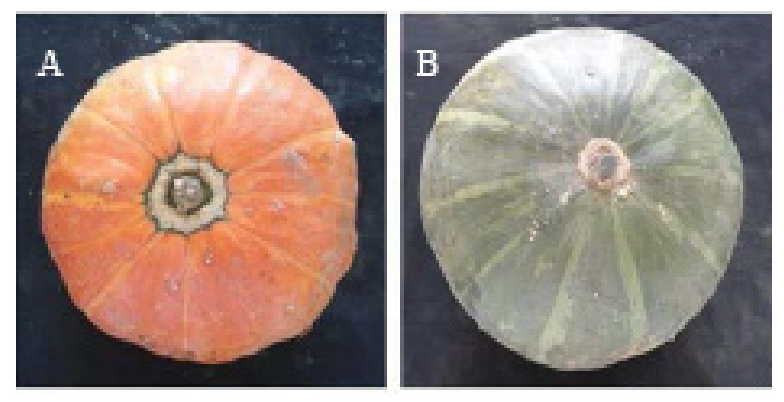

Figure 1. A. Paternal parent (06820-1) with orange rind. B. Maternal parent (98-2-351) with gray rind.

The JoinMap version 3.0 software (Stam, 1993; Van Ooijen and Voorrips, 2001) was used to construct the linkage map with logarithm (base 10) of odds scores of 4.0-6.0 (Kosambi, 1994). Mendelian segregation was tested using the chi-squared test performed under the "Locus Genotypic Frequency" command. The markers were sorted based on the chi-squared test at a P value of $<0.05$. Mapchart 2.1 was used for constructing the maps (Voorrips, 2002).

\section{RESULTS}

Of the 1692 primer pairs (including 1386 SSRs, 256 AFLPs, and 50 RAPDs) screened for polymorphisms between the parental lines '98-2-351' and '06820-1', only 57 SSRs, 21 AFLPs, and 3 RAPDs were found to be polymorphic. A total of 82 polymorphic markers, containing 81 molecular markers and 1 phenotypic marker, were grouped into the 10 linkage groups (LGs) of C. maxima with 4.0-6.0 for logarithm (base 10) of odds values. The total length of the genetic map was $991.5 \mathrm{cM}$ and the average distance between adjacent markers was $12.1 \mathrm{cM}$ (Table 1 and Figure 2). The 20 LGs of $C$. maxima varied in map length, the number of markers, and marker density (Table 1). LG length varied from $20.4 \mathrm{cM}$ in LG5 to 123.2 cM in LG6. The number of markers in the 20 LGs ranged from 2 in LG17, LG18, LG19, and LG20 to 11 in LG1. Two SSR markers, 'PU078072' and 'PU013839', were found to be tightly linked to the locus $R c$ at a distance of 5.9 and $14.5 \mathrm{cM}$ in LG5, respectively (Figure 2).

Table 1. Characteristics of 20 linkage groups of genetic maps of Cucurbita maxima.

\begin{tabular}{lccr}
\hline Linkage groups & Map length (cM) & Number of markers & Average marker density (cM) \\
\hline LG1 & 70.1 & 11 & 6.4 \\
LG2 & 28.2 & 4 & 7.1 \\
LG3 & 61.3 & 6 & 10.2 \\
LG4 & 34.1 & 3 & 11.4 \\
LG5 & 20.4 & 3 & 6.8 \\
LG6 & 123.2 & 8 & 15.4 \\
LG7 & 48.0 & 3 & 16.0 \\
LG8 & 52.4 & 3 & 17.5 \\
LG9 & 79.1 & 6 & 13.2 \\
LG10 & 35.1 & 3 & 11.7 \\
LG11 & 72.1 & 5 & 14.4 \\
LG12 & 57.8 & 5 & 11.6 \\
LG13 & 44.7 & 4 & 11.2 \\
LG14 & 60.0 & 4 & 15.0 \\
LG15 & 26.0 & 3 & 8.7 \\
LG16 & 28.0 & 2 & 9.3 \\
LG17 & 32.8 & 2 & 16.4 \\
LG18 & 45.4 & 2 & 18.7 \\
LG19 & 35.9 & 2 & 18.5 \\
LG20 & 36.9 & 82 & 12.1 \\
Total & 991.5 & & \\
\hline
\end{tabular}



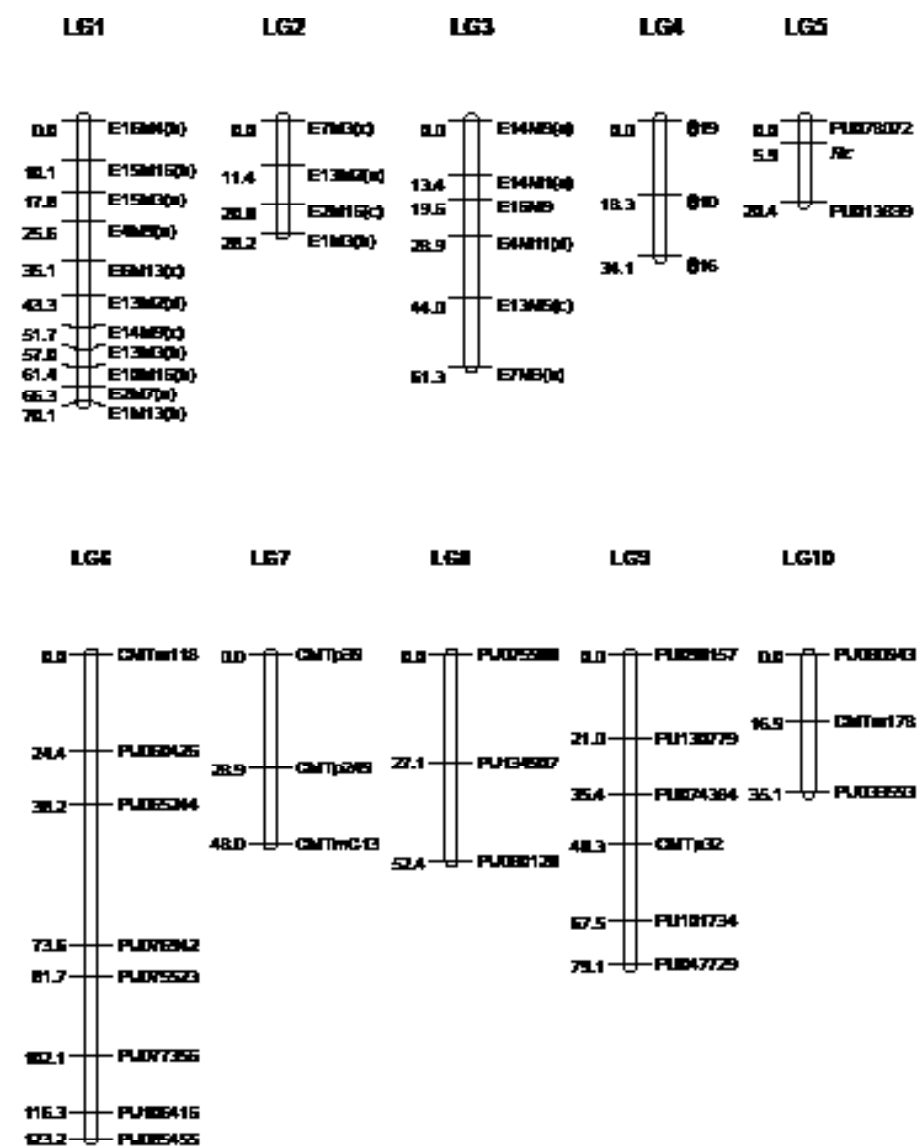

Figure 2. Genetic linkage map of Cucurbita maxima showing distribution of AFLPs, RAPDs, SSRs, and 1 phenotypic marker. The phenotypic marker (rind color) is denoted by ' $R c^{\prime}$ ' in italics.

\section{DISCUSSION}

All published genetic map populations for the genus Cucurbita were $\mathrm{F}_{2}$ or $\mathrm{BC}_{1}$ population and the number of LGs in all published genetic maps of the genus Cucurbita ranged from 5-28 (Weeden and Robinson, 1986; Lee et al., 1995; Brown and Myers, 2002; Zraidi et al., 2007; Gong et al., 2008a,b; Esteras et al., 2012). The haploid chromosome number of genus Cucurbita is 20, but only the LG number of 2 maps was in accordance with the haploid chromosome number among all published genetic maps (Gong et al., 2008a,b). In the present study, the number of LGs on the genetic map was 20, which aligned the LG with the corresponding chromosome when the 'anchor' markers were further developed and integrated into the present map. This may facilitate the genes or loci controlling economically important traits to be located in the corresponding chromosome.

The length of the newly developed genetic map of 82 markers, including 57 SSRs, 21 AFLPs, 3 RAPDs, and 1 phenotypic marker, was $991.5 \mathrm{cM}$, which was shorter than the 
maps described by Brown and Myers (2002), Zraidi et al. (2007), Gong et al. (2008a,b), and Esteras et al. (2012), where map lengths were 1445-2234 cM. The observation of different genetic map lengths in several studies contributed to the type and number of markers, different mapping populations, individual numbers, software, and scoring errors used for analyses. However, the addition of markers, particularly 'anchor' markers, is important for improving the map of $C$. maxima developed in the present study.

\section{ACKNOWLEDGMENTS}

Research supported by a grant from the Research Fund for the Public Welfare Industry (Agricultural) Research Special Foundation of the Agricultural Ministry of China (\#201303112) and the Foundation of Heilongjiang Educational Committee (\#12531043).

\section{REFERENCES}

Brown RN and Myers JR (2002). A genetic map of squash (Cucurbita sp.) with randomly amplified polymorphic DNA markers and morphological markers. J. Am. Soc. Hort. Sci. 127: 568-575.

Esquinas-Alcazar JT and Gulick PJ (1983). Genetic Resources of Cucurbitaceae. IBPGR, Rome.

Esteras C, Gómez P, Monforte AJ, Blanca J, et al. (2012). High-throughput SNP genotyping in Cucurbita pepo for map construction and quantitative trait loci mapping. BMC Genomics 13: 80.

Ge Y, Ramchiary N, Wang T, Liang C, et al. (2011). Development and linkage mapping of unigene-derived microsatellite markers in Brassica rapa L. Breed. Sci. 61: 160-167.

Ge Y, Wang T, Wang N, Wang Z, et al. (2012). Genetic mapping and localization of quantitative trait loci for chlorophyll content in Chinese cabbage (Brassica rapa ssp. pekinensis). Sci. Hortic. 147: 42-48.

Gong L, Stift G, Kofler R, Pachner M, et al. (2008a). Microsatellites for the genus Cucurbita and an SSR-based genetic linkage map of Cucurbita pepo L. Theor. Appl. Genet. 117: 37-48.

Gong L, Pachner M, Kalai K and Lelly T (2008b). SSR-based genetic linkage map of Cucurbita moschata and its synteny with Cucurbita pepo. Genome 51: 878-887.

Kosambi DD (1944). The estimation of map distance from recombination values. Ann. Eugen. 12: 172-175.

Lee YH, Jeon HJ, Hong KH and Kim BD (1995). Use of random amplified polymorphic DNA for linkage group analysis in an interspecific cross hybrid $\mathrm{F}_{2}$ generation of Cucurbita. J. Kor. Soc. Hortic. Sci. 36: 323-330.

Li X (2014). Gene mapping of peel and flesh color of pumpkin (Cucurbita maxima). Dissertation thesis, Northeast Agricultural University, Heilongjiang.

Robinson RW and Decker-Walters DS (1997). Cucurbits. CAB International, New York.

Sanjur OI, Piperno DR, Andres TC and Wessel-Beaver L (2002). Phylogenetic relationships among domesticated and wild species of Cucurbita (Cucurbitaceae) inferred from a mitochondrial gene: Implications for crop plant evolution and areas of origin. Proc. Natl. Acad. Sci. U. S. A. 99: 535-540.

Stam P (1993). Construction of integrated genetic linkage maps by means of a new computer package: JoinMap. Plant $J$. 3: 739-744.

Van Ooijen JW and Voorrips RE (2001). JoinMap Version 3.0: software for the calculation of genetic linkage maps. Plant Res Intl, Wageningen.

Voorrips RE (2002). MapChart: software for the graphical presentation of linkage maps and QTLs. J. Hered. 93: 77-78.

Weeden NF and Robinson RW (1986). Allozyme segregation ratios in the interspecific cross Cucurbita maxima $\mathrm{x}$ C. equadorensis suggest that hybrid breakdown is not caused by minor alterations in chromosome structure. Genetics 114: 593-609.

Whitaker and Davis GN (1962). Cucurbits, Botany, Cultivation and Utilization. Leonard Hill, London.

Zraidi A, Stift G, Pachner M, Shojaeiyan A, et al. (2007). A consensus map for Cucurbita pepo. Mol. Breed. 20: 375-388. 